\title{
强冲击作用下金属界面物质喷射与混合问题数值 模拟和理论研究
}

\author{
王裴 $1^{*}$, 何安民 ${ }^{1}$, 郡建立 $^{2}$, 孙海权 $^{1}$, 陈大伟 ${ }^{1}$, 刘文斌 1,3 , 刘 ${ }^{1}{ }^{1}$ \\ 1. 北京应用物理与计算数学研究所, 北京 100094 ; \\ 2. 北京理工大学爆炸科学与技术国家重点实验室, 北京 100081; \\ 3. 中国工程物理研究院研究生院, 北京 100088 \\ *联系人, E-mail: wangpei@iapcm.ac.cn \\ 收稿日期：2018-04-11; 接受日期：2018-06-06; 网络出版日期：2018-07-20 \\ 国家自然科学基金(编号: U1530216)和科学挑战专题(编号: TZ2016001)资助项目
}

\begin{abstract}
摘要当强冲击波从金属自由面反射时通常会发生微喷射现象, 气体环境下, 喷射颗粒将与气体相互作用而形成 物质混合. 微喷射与混合是战略武器研究中的关键和难点问题, 亦是冲击压缩科学与工程领域研究的前沿和热点 问题之一。作者与合作者长期从事金属微喷射与混合问题理论和数值模拟研究，取得了一系列研究进展. 本篇评 述简要介绍我们围绕微喷射物理机制与影响因素、理论建模以及气粒混合问题开展的数值模拟和理论研究工 作, 以及取得的研究成果. 主要包括以下几个方面: (1) 微射流喷射数值模拟与理论建模; (2) 微层裂破碎数值模拟 与机理分析; (3) 微喷混合数值模拟与动力学规律.
\end{abstract}

关键词微喷射, 混合, 微射流, 微层裂

PACS: 47.15.Uv, 62.50.Ef, 47.11.-j

\section{1 引言}

对于受到冲击加载的金属材料, 当冲击波从金属 自由面或者金属/气体界面卸载时, 金属表面会发生破 坏或破碎，并产生高速颗粒态物质脱离基体而向外喷 射, 这一动力学现象称为微喷射. 在气体环境下, 金属/ 气体界面位置产生的喷射颗粒将与气体相互作用而形 成物质混合. 微喷射及其与气体的混合是诸多冲击压 缩工程领域中非常关注的重点和难点问题, 在战略武
器研究中占有至关重要的地位. 此外, 微喷射和混合 研究还涉及材料冲击相变、动态损伤和破坏、多相流 动以及颗粒气动变形破碎等大量的前沿基础性科学问 题, 研究成果对于促进和丰富材料冲击响应和多相流 动的理论研究也具有重要学术价值.

微喷射现象于 20 世纪 50 年代即被发现 ${ }^{[1]}$ ，因其重 要的应用背景, 一直受到各核大国武器物理实验室的 高度重视。由于微喷射具有喷射过程时间短 $(<100 \mathrm{~ns})$ 、喷射颗粒速度高 $(\mathrm{km} / \mathrm{s})$ 、以及颗粒尺度小

引用格式: 王裴, 何安民, 邵建立, 等. 强冲击作用下金属界面物质喷射与混合问题数值模拟和理论研究. 中国科学: 物理学 力学 天文学, 2018, 48: 094608 Wang P, He A M, Shao J L, et al. Numerical and theoretical investigations of shock-induced material ejection and ejecta-gas mixing (in Chinese). Sci Sin-Phys Mech Astron, 2018, 48: 094608, doi: 10.1360/SSPMA2018-00116 
$(\mu \mathrm{m})$ 等特点, 给实验的精细观测带来了极大困难. 数十 年来, 各国均花费了较大的人力、物力, 推动微喷射定 量测试技术的不断发展，从早期的单一测试技术一 Asay膜 ${ }^{[2-4]}$ ，逐步发展到压电探针 ${ }^{[5]} 、 \mathrm{X}$ 光 ${ }^{[6]}$ 、高分辨 率全息 ${ }^{[7]}$ 、高速摄影 ${ }^{[8]}$ 和质子照相 ${ }^{[9]}$ 等多种测试技术 的联合观测. 大量的实验研究表明 ${ }^{[10-24]}$, 微喷射是一种 极其复杂的界面动力学行为, 主要影响因素包括表面缺 陷结构、冲击加载压力与波形、材料强度以及卸载熔 化等. 在不断总结实验规律的基础上，研究人员逐渐发 现冲击波与表面微结构缺陷相互作用形成的微射流 ${ }^{[2,4]}$ 以及衰减冲击波加载下近表面熔化物质动态拉伸破坏 引起的微层裂 ${ }^{[17]}$ 是造成物质喷射的两种主导机制.

随着对微喷射认识的不断深入以及工程研究的迫 切需要, 充气条件下的微喷混合问题亦备受关注. 1989 年, 法国Elias等人 ${ }^{[25]}$ 率先开展了微喷混合实验, 发现气 体环境下喷射物的运动受到了明显抑制，最终在金属 表面形成稳定厚度的混合层. 随后，俄、美等国的研 究人员陆续开展了相关实验 ${ }^{[26-28]}$, 获得了微喷物质与 气体混合的基本物理图像、混合区的头部运动规律和 质量-速度分布等重要信息, 为细致研究微喷颗粒与气 体的混合机理和动力学规律提供了重要的实验依据.

进入 21 世纪之后, 随着计算机技术的飞速发展, 数 值模拟成为微喷射和混合研究中不可或缺的重要技术 手段 ${ }^{[29-35]}$. 借助这一强大工具, 我们一方面可以获得实 验上无法观测的物质喷射与混合的动力学过程、解读 实验结果并揭示其物理机理；另一方面可以灵活针对 单一影响因素开展序列化的数值模拟, 为理论建模提 供定量化的规律认识. 在微喷射数值模拟研究方面, 因其本身属于多尺度、多物理的动力学问题，综合使 用不同尺度数值模拟技术，包括微观分子动力学和宏 观流体动力学，对同一现象开展多尺度联合模拟与多 角度分析是该问题的最大研究特色. 正是基于不同尺 度数值模拟，通过无量纲参数分析，美国Los Alamos 国家实验室 ( $\mathrm{LANL}$ ) 的研究人员基于流体介质 Richtmyer-Meshkov界面不稳定性(RMI)理论，建立了 描述熔化条件下微射流喷射总量和最大速度的理论模 型 ${ }^{[36-38]}$. 对于混合问题, 由于微喷颗粒与气体的混合过 程属于极端条件下的两相流动问题，并且还包含颗粒 气动破碎、化学反应等复杂物理化学过程, 使得混合 数值模拟研究具有极大的挑战性, 目前尚处于起步阶 段 ${ }^{[39,40]}$.
本篇评述将简要介绍十余年来，作者与合作者基 于自主研制的分子动力学(MD)、光滑粒子流体动力 学 $(\mathrm{SPH}) 、 气$ 体-颗粒两相流程序, 围绕微喷射物理机 制、理论建模以及混合问题开展的一系列数值模拟和 理论研究工作, 包括: (1) 在微射流喷射方面, 基于不同 尺度数值模拟, 细致分析了微射流的形成机制, 以及表 面缺陷、材料强度和卸载熔化等因素的对喷射状态的 影响特征和机理; 基于数值模拟获得的规律性认识, 结 合理想弹塑性介质RMI理论，建立了强度介质微射流 喷射理论模型; 采用MD模拟, 给出了微射流动态破碎 物理过程及其阶段性演化特征，获得了破碎颗粒尺寸 分布规律，并揭示了不同粒径分布形式的物理根源; (2) 在微层裂破碎方面，给出了材料损伤破坏微观的 演化过程和熔化前后物质破坏模型的转化机制，获得 了熔化效应对材料自由面速度历史和拉伸断裂强度的 影响特征; (3) 建立了微喷混合数值模拟程序, 获得了 平面以及内爆压缩柱面汇聚流场下微喷颗粒与气体混 合演化的基本图像，以及初始气体压力和微喷颗粒对 气体流场反馈效应的影响特征，揭示了高速金属液滴 气动破碎这一影响混合状态的关键物理因素.

\section{2 微射流喷射数值模拟和理论建模}

20 世纪70年代, Asay等人 ${ }^{[2-4]}$ 对金属铅、铝的微喷 射行为进行了实验研究, 发现了表面粗糙度是影响喷 射总量的重要因素. 由此, Asay首先提出了与样品表 面几何缺陷相关的微射流喷射机制，即金属表面在机 加工过程中形成的微米尺度沟槽在强冲击作用下被激 活而形成微射流，微射流在后续演化过程中断裂破碎， 最终形成高速喷射颗粒. 此后的实验研究还发现, 除了 表面粗䊁度，冲击波加载速率、卸载熔化等因素都会 对喷射状态产生显著影响，但实验上无法给出这些因 素对喷射状态的影响机理. 为此，我们对冲击作用下 含表面缺陷金属的微射流喷射过程开展了不同尺度数 值模拟, 分析了微射流的形成机制, 以及沟槽角度、冲 击加载速率、卸载熔化等因素对喷射状态的影响特征 和机理.

\section{1 微射流喷射形成机制与影响因素}

利用自主研制的SPH数值模拟程序，我们对方波 持续冲击作用下，含表面 $\mathrm{V}$ 形沟槽金属铝的微喷射过 
程进行了数值模拟 ${ }^{[41]} ，$ 给出了冲击波与沟槽表面相互 作用形成微射流的动力学机制: 当冲击波从沟槽斜面 反射后，斜面附近物质获得几乎与斜面垂直的速度， 向沟槽中心运动并在沟槽中心线附近碰撞形成高速射 流，如图1所示. 数值模拟给出微射流总质量、头部速 度、质量-速度分布等结果均与实验符合较好, 确认了 高速喷射物的微射流形成机制. 此外, 我们考察了加载 波前沿宽度 ${ }^{[42]}$ 和沟槽夹角 ${ }^{[43]}$ 对微射流状态的影响，发 现：(1) 随加载波前沿宽度的增加，射流质量和头部速 度均减小，同时低速喷射物所占比例增大；(2) 射流质 量在沟槽半角为 $45^{\circ}$ 附近达到最大, 随着角度的增加或 减小射流质量均减小; 最大喷射速度随沟槽角度的增 加近似线性减小, 与实验规律一致. 进一步, 我们通过 追踪微射流的物质来源和运动轨迹，分析了二者影响 微射流状态的动力学机理.

对于卸载熔化对金属微射流状态的影响，由于连 续介质理论尚无法细致描述卸载熔化物理过程, 我们 基于自主研制的大规模并行MD程序，通过引入能够 反映金属高压熔化的嵌入原子势，对含表面沟槽金属 铝的微射流喷射问题进行了系列模拟 ${ }^{[31]}$. 统计分析了 微射流质量分布、头部速度以及喷射系数随波后粒子 速度(冲击压力)的变化规律. 研究结果表明: 熔化前后, 微射流头部速度与波后粒子速度始终保持较好的线性 增长关系; 而喷射系数在熔化前随波后粒子速度线性 增长, 当卸载熔化发生后, 喷射系数则迅速增加. 这些

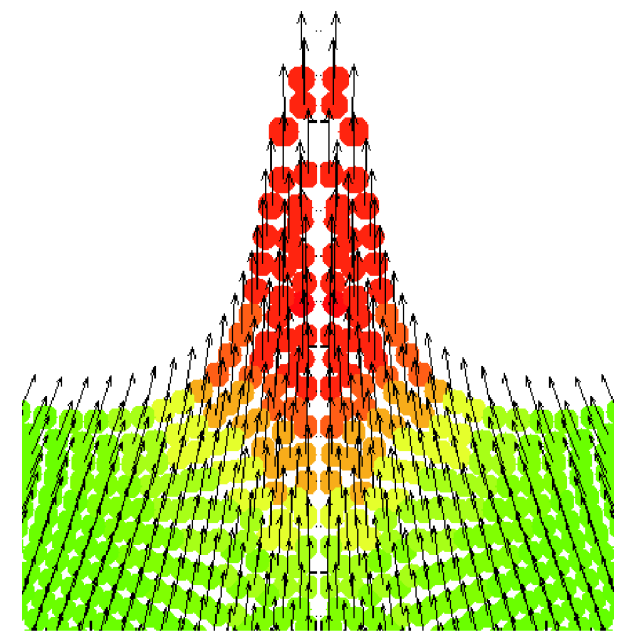

图 1 (网络版彩图)射流形成过程中物质速度矢量分布 Figure 1 (Color online)Velocity vector distribution of SPH particles in microjet.
变化规律与相关实验测量结果基本一致. 结合微结构 和力学量分析，揭示了熔化后微喷射总量显著增加的 物理机制，即熔化后微射流周边区域的物质流动性明 显加强，使得微射流根部相对熔化前明显变粗，进而 造成喷射总量的显著增加, 如图2所示.

\section{2 微射流喷射理论模型}

在实验和数值模拟均明确了高速低密度喷射物的 微射流形成机制后，微喷射理论研究的目标便自然聚 焦到建立一种可以描述微射流总量和最大速度随着材 料性质、表面缺陷状态和加载条件变化的理论模型. 2012年，美国LANL研究人员将微射流视为一种特殊 的金属/真空界面RMI现象，利用流体介质RMI扰动增 长理论，建立了描述熔化条件下微射流喷射总量和最 大速度的理论模型 ${ }^{[36-38]}$. 需要指出的是, 该模型是建立 在材料完全熔化的基本假设之上，而根据强度介质 RMI理论，未熔化材料同样会发生界面塑性流动失稳. 在此情况下，虽然强度效应会抑制界面不稳定性的发 展，但在特定状态下RM尖钉会持续增长，最终断裂破 碎而形成物质喷射 ${ }^{[29]}$. 因此, 发展强度介质微射流喷射 总量和最大速度量化描述方法, 是构建完整的缺陷喷 射理论亟需解决的重要问题.

围绕冲击作用下强度介质微射流缺陷喷射理论建 模，我们采用自行研制的二维多介质弹塑性欧拉流体 动力学程序—- $\mathrm{MEPH}^{[44]}$, 对强度介质界面不稳定性 和物质喷射开展了数值模拟，着重分析了强度效应 对界面扰动增长和物质喷射的影响规律. 我们发现: (1) 强度介质的RM尖钉和气泡呈现非对称演化特征, 即气泡发展总是受到抑制，振幅趋于饱和，而尖钉增 长只是在特定条件下受到抑制，否则就会持续增长,

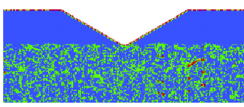

(a) $t=4.0 \mathrm{ps}$

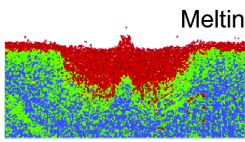

(c) $t=6.0 \mathrm{ps}$

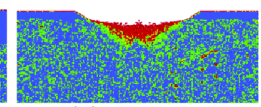

(b) $t=4.7 \mathrm{ps}$

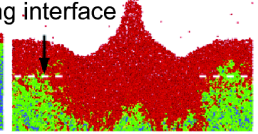

(d) $t=9.0 \mathrm{ps}$

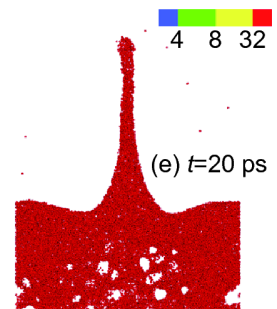

图 2 (网络版彩图)卸载熔化样品的微射流演化图像 ${ }^{[31]}$

Figure 2 (Color online) Microscopic morphology evolution of microjets formation after release melting. Reproduced from ref. [31], with the permission of AIP Publishing. 
直至发生断裂破碎而形成物质喷射; (2) 当尖钉持续增 长形成物质喷射时，喷射总量在气泡振幅饱和时趋于 恒定值，并且随着气泡饱和振幅的增加而增大，表明 强度介质喷射总量与气泡饱和振幅之间存在一定的内 在联系.

基于以上规律认识，结合气泡饱和时刻样品的表 面失稳图像(图3)，通过理论推导，我们给出了喷射物 质总量对气泡饱和振幅的依赖关系. 在此基础上，利 用强度介质RMI理论研究成果, 包括RM尖钉增长与材 料强度的依赖关系 ${ }^{[45,46]}$ 、理想弹塑性介质气泡饱和振 幅的解析表达式 ${ }^{[47]}$ ，建立了强度介质微射流喷射形成 的临界条件、喷射总量和最大喷射速度的理论模 型 ${ }^{[48,49]}$. 该模型与LANL纯流体模型 ${ }^{[38]}$ 一起，构成了完 整的描述喷射状态(总量、最大速度)随着材料性质、 表面扰动状态和加载压力变化的微射流喷射理论 模型.

\section{3 微射流动态破碎与颗粒尺寸统计分布}

微射流形成后, 在速度梯度作用下不断拉伸, 最终 断裂破碎, 形成破碎颗粒. 微射流断裂破碎颗粒尺寸分 布是微喷射物理建模的主要内容之一，同时也是微喷 混合数值模拟的输入条件以及影响混合演化过程的重 要物理因素. 由于微射流断裂破碎是一个典型的多尺 度、跨学科问题，对理论研究和基于连续介质理论的 流体动力学模拟提出了极大的挑战. 2012年，法国 CEA研究人员Durand等人将MD方法应用于微射流破 碎颗粒尺度研究，给出了微喷射动态破碎过程的阶段 性演化特征以及不同破碎阶段的颗粒粒径分布 ${ }^{[33,50]}$.

基于自主研制的大规模并行MD程序，我们对金 属表面沟槽微射流动态破碎和颗粒尺寸分布进行了数

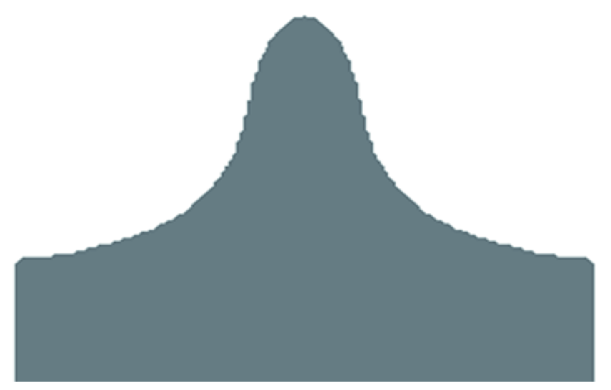

图 3 气泡振幅饱和时强度介质界面失稳图像

Figure 3 Snapshot of RMI for solid $\mathrm{Pb}$ at the instant when bubble have saturated.
值模拟分析，综合考察了冲击强度、沟槽尺寸等因素 的影响 ${ }^{[1,52]}$. 数值模拟结果显示, 在不同冲击压力和沟 槽尺寸条件下，微射流的破碎过程均可以划分为三个 不同阶段(图4): (1) 射流内部出现随机分布的孔隙结 构，孔隙逐渐增长、贯通，导致初始密实片状射流体 转化为由条状碎片组成的二维网状结构; (2) 网状结 构在速度梯度作用下继续拉伸、解体并释放出大小不 一的条状碎片; (3) 体积较大的条状碎片继续破碎成更 小尺度的破碎物, 由于表面张力效应, 小尺度破碎物最 终全部转化为球形液态颗粒. 微观模拟获得的微射流 破碎阶段性演化特征与高分辨率全息实验观测结果一 致 ${ }^{[28]}$

在破碎颗粒尺寸统计分布规律方面，我们发现微 射流完全破碎后的粒径统计分布由几种不同分布形式 组合而成, 即较大尺度颗粒满足双指数分布, 而较小尺 度颗粒服从包含不同特征常数幂律分布，如图5所示. 较大尺度颗粒的双指数分布与微射流破碎颗粒尺寸的 高分辨率全息 ${ }^{[28]}$ 和软回收 ${ }^{[53]}$ 实验观测结果相吻合. 进 一步，基于数值模拟得到的微射流破碎动力学演化特 征，结合动态破碎唯象理论，我们分析了不同分布形 式的物理根源. 其中, 幂律分布符合渗流理论结果, 而 双指数分布则可以利用包含统计非均匀性的几何统计 破碎理论解释. 在双指数分布中, 包含了两个特征尺寸 参数. 通过追踪不同特征尺寸颗粒的物质来源, 我们发 现造成系统包含两个特征尺寸的原因在于碎片网络结 构解体后所释放的条状碎片空间取向的不均匀性; 由 于相对于射流拉伸方向，不同取向条状碎片承受的拉 伸速率不同, 使得破碎后的颗粒具有不同特征尺寸.

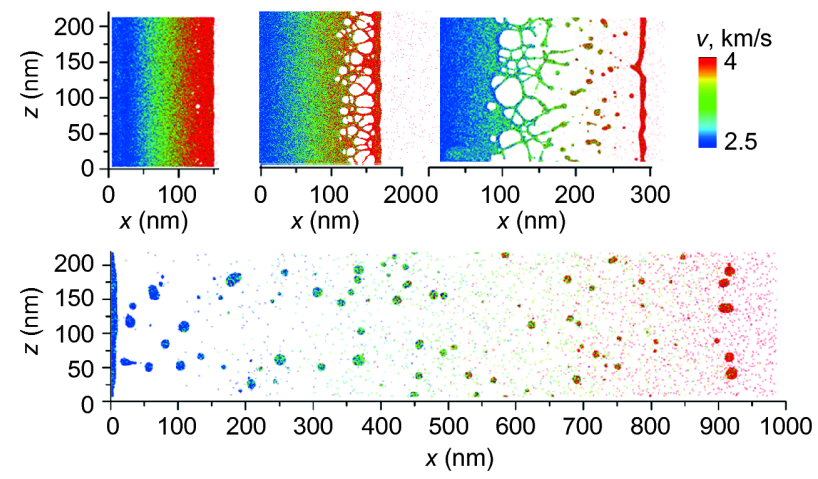

图 4 (网络版彩图)微射流断裂破碎MD模拟图像 ${ }^{[52]}$

Figure 4 (Color online) Side views of microjet breakup and ejecta production. Reproduced from ref. [52], with the permission of IOP Publishing. 


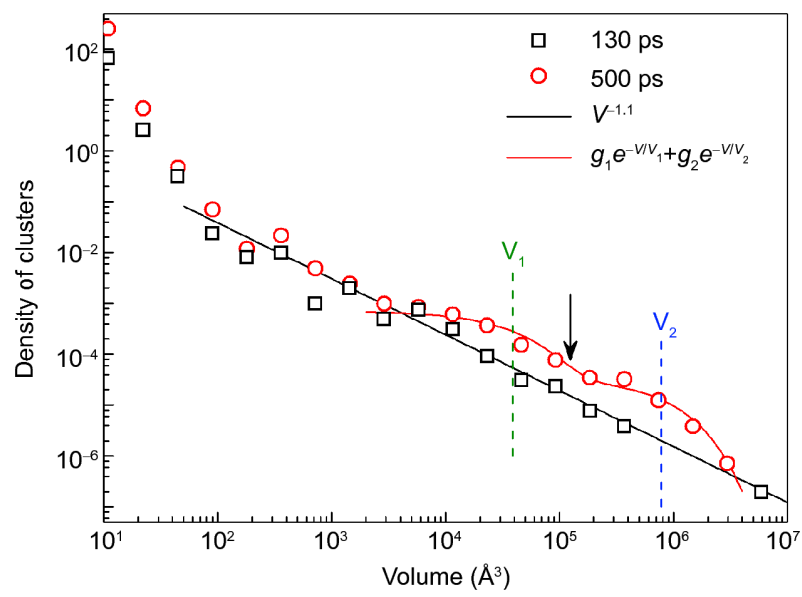

图 5 (网络版彩图)微射流不同破碎阶段的碎片数目-体积 分布 ${ }^{[52]}$

Figure 5 (Color online) Log-log volume distributions of fragment at different breakup stages. Reproduced from ref. [52], with the permission of IOP Publishing.

为考察应变率效应对射流动态破碎过程和粒径分 布的影响，我们还构造了速度梯度作用下液态金属薄 膜动态破碎的简化模型，并开展了微观模拟分析．研 究结果表明：即使在相对较低应变率作用下，液态金 属薄膜的动态破碎过程同样可以划分为碎片网络结构 形成、网络结构解体以及条状碎片二次破碎三个特征 阶段; 当薄膜完全破碎成球形颗粒之后, 破碎颗粒粒径 分布同样由小尺度颗粒的幂律和大尺度颗粒的双指数 分布组成，并且双指数分布中的特征尺寸参数随着拉 伸应变率的降低而增大 ${ }^{[54]}$.

\section{3 微层裂破碎数值模拟与机理分析}

除了冲击波与表面缺陷相互作用形成的微射流喷 射，当加载波为衰减冲击波(爆轰或高功率脉冲激光直 接驱动的典型波形)且材料熔化后, 近表面物质在入射 稀疏波与自由面反射稀疏波形成的动态拉伸力作用下 将破碎成大量的细小液态颗粒而形成物质喷射，这种 熔化条件下近表面物质的动态拉伸破坏现象又称为微 层裂 ${ }^{[17]}$. 由于微层裂物质的密度较微射流大幅增加, 且 破碎颗粒间的速度差异小，给实验的定量观测造成极 大的困难, 对其物理机制和规律性认识还很缺乏.

\section{1 微层裂动力学与破坏形态}

为获得金属微层裂破碎的动态物理过程，尤其是
熔化前后材料破坏形态的差异，我们采用MD方法数 值模拟了三角波加载下金属铝动态破坏行为，分析了 样品内部微结构的演化过程和熔化前后物质破坏形态 的差异 ${ }^{[32]}$. 结果表明: 较低冲击压力下(熔化前), 近表 面区域物质在卸载稀疏波形成的双向拉伸作用下出现 孔洞形核、生长与贯通、最终导致了表面固态层裂片 出现，见图6; 而较高冲击压力下(熔化后), 当三角波从 金属自由面反射时，首先观察到表面出现逃逸原子或 团簇，之后孔洞在近表面区域成核、长大，并且随着 卸载过程继续不断出现新的孔洞成核, 最终形成了大 面积的液态空穴化和破碎区域, 见图7.

为说明材料近表面物质的破坏程度随三角波强度
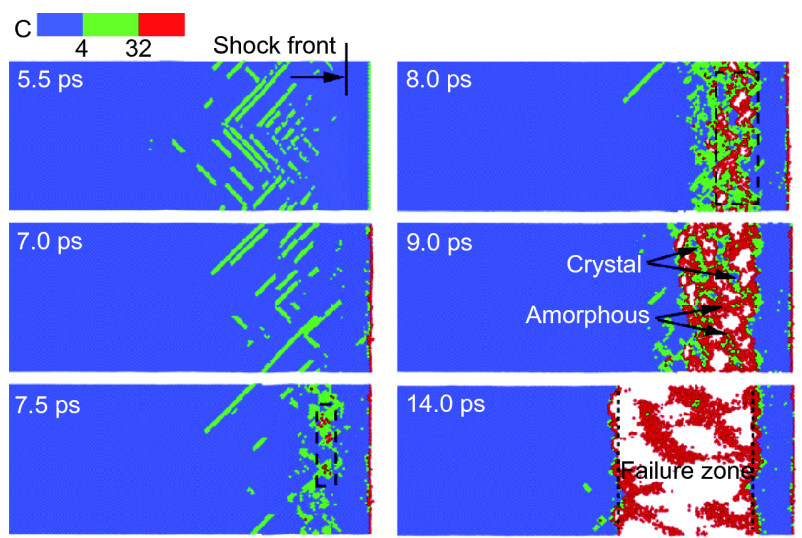

图 6 (网络版彩图)三角波加载下，固态金属 $\mathrm{Al}$ 层裂微观动 力学过程 ${ }^{[32]}$

Figure 6 (Color online) Microscopic morphology evolution of spallation in solid Al. Reproduced from ref. [32], with the permission of AIP Publishing.
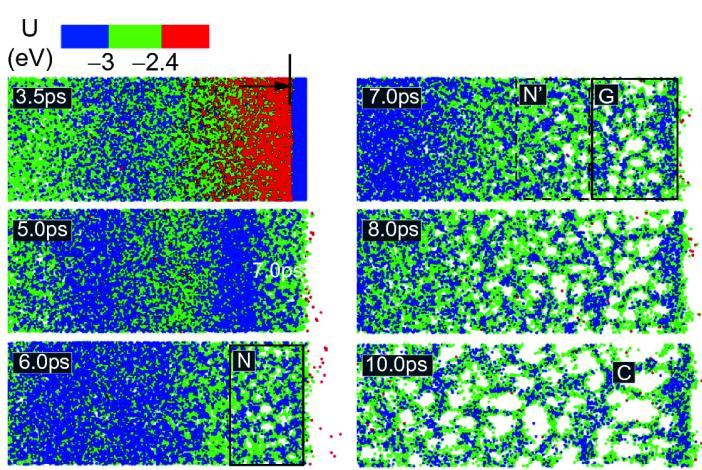

图 7 (网络版彩图)三角波加载下，熔化金属 $\mathrm{Al}$ 层裂微观动 力学过程 ${ }^{[32]}$

Figure 7 (Color online) Microscopic morphology evolution of spallation in liquid Al. Reproduced from ref. [32], with the permission of AIP Publishing. 
的变化，我们统计了不同碰撞速度情况下材料的破坏 深度(即发生破碎的物质对应材料初始状态时的厚度) 的变化. 模拟结果显示, 熔化前破坏深度随碰撞速度近 似满足线性增长关系; 而当卸载熔化发生后, 材料的破 坏深度开始逐渐偏离线性关系，并且随着碰撞速度的 继续增加，破坏深度呈现明显的非线性增长，如图8 所示.

材料在熔化前后破坏动力学过程和损伤状态的差 异，在自由面速度历史曲线上具有清晰的反映信号. 图9给出了不同碰撞速度下的样品自由面速度历史. 可以看出, 碰撞速度较低时 $(1.0-2.0 \mathrm{~km} / \mathrm{s})$ 时, 金属自由 面出现明显的振荡信号，并恰好对应固态层裂片的产 生; 而当碰撞速度较高 $(3.5 \mathrm{~km} / \mathrm{s}-6.0 \mathrm{~km} / \mathrm{s})$ 时, 只观察 到自由面峰值速度的衰减，不再有任何振荡信号。显 然，后者是由于卸载熔化导致了大面积空穴化与破坏 区的形成，固态下出现的断裂面信号难以在这种状态 下形成和传播.

\section{2 微射流与微层裂耦合作用下物质喷射状态}

一般而言，爆轰或高功率脉冲激光加载下机加工 样品的物质喷射状态是由微射流与微层裂机制共同作 用的结果. 针对这一问题，结合实际样品表面形貌特 征，我们开展衰减冲击加载下金属微喷射不同尺度数 值模拟研究，给出了微喷射的形成机制以及喷射物的 空间分布特征 ${ }^{[5]}$. 图10给出了方波和强稀疏三角波加

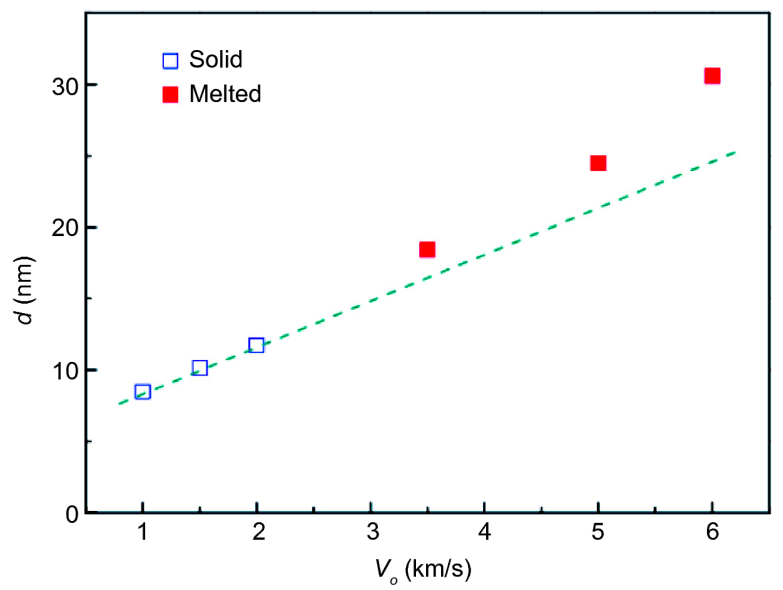

图 8 (网络版彩图)三角波加载下，金属铝近表面破坏深度 随碰撞速度的变化 ${ }^{[32]}$

Figure 8 (Color online) Variation of failure depth with the impact velocity. Reproduced from ref. [32], with the permission of AIP Publishing.

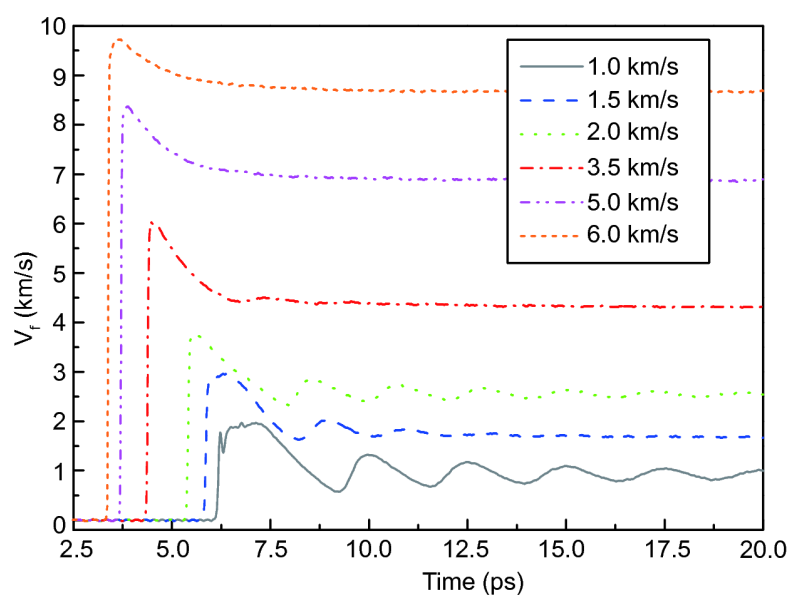

图 9 (网络版彩图)不同撞击速度下，样品自由面速度历 史 $^{[32]}$

Figure 9 (Color online) Evolution of free-surface velocity with time for different impact velocities. Reproduced from ref. [32], with the permission of AIP Publishing.

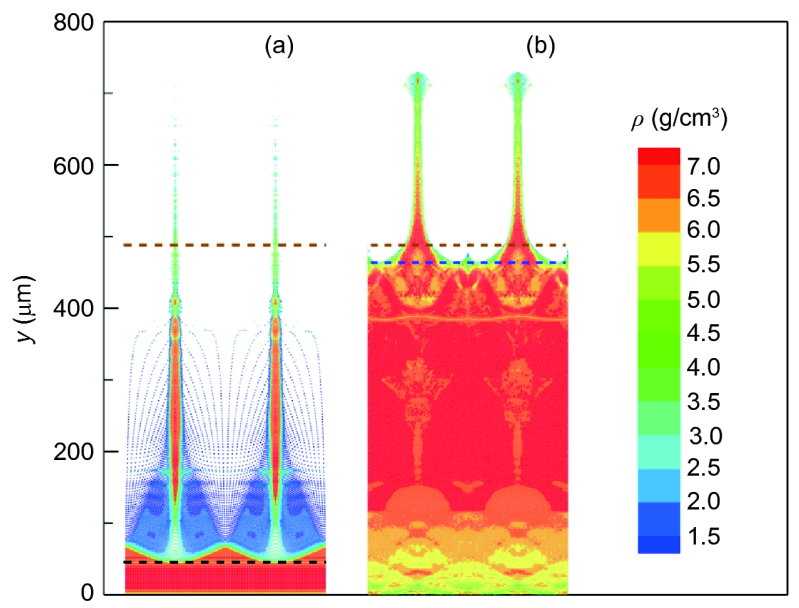

图 10 (网络版彩图)三角波(a)与方波(b)加载下的微喷射 图像

Figure 10 (Color online) Ejecta morphologies under unsupported (a) and supported (b) shocks.

载下含表面沟槽金属微喷射SPH数值模拟图像. 可以 看出，与方波加载下喷射物质主要表现为沟槽射流不 同，在三角波加载下喷射体由源于沟槽的高速聚能射 流以及沟槽间复杂波系作用形成的连续分布低密度破 碎区组成, 并且喷射物质量向沟槽中间线集中. 数值模 拟获得的密度分布特征与质子照相的实验结果非常相 似. 对于喷射量, 数值模拟表明: 方波冲击条件下, 截止 气泡底部, 喷射量大体与初始缺陷质量相当; 而强稀疏 三角波加载下, 高速射流量减少, 但由于稀疏作用引起 
低速破碎区显著增大了喷射总量, 截断位置推后, 喷射 总量显著增加.

此外，基于MD模拟，我们分析了三角波衰减速率 对喷射状态的影响，重点考察了从弱稀疏至强稀疏下 微射流机制的变化和微层裂破碎状态，发现：在弱稀疏 情况(界面完整), 头部速度对波形依赖较小, 波形主要导 致界面的回拉并使界面形状发生改变, 由此可导致微射 流质量倍数增加. 而在强稀疏条件下，伴随能量损耗微 射流发展受到抑制，同时产生大面积微层裂区域，这使 得三角波加载下的喷射场呈现光滑增长和平台振荡阶 段, 相应的速度分布呈现两段式的线性分布 ${ }^{[6]}$.

\section{4 微喷混合数值模拟与动力学规律}

由于微喷射形成以及微喷射物质与气体的混合是 一个跨尺度、多物理的复杂动力学过程. 考虑到微喷 射过程时间尺度(百纳秒)远小于其后续的混合过程(数 十微秒), 我们对二者进行解耦, 将材料在真空条件下 的微喷射状态作为混合的初值条件, 采用气体-颗粒两 相流颗粒轨道模型模拟微喷颗粒在气体中的运动和混 合过程. 以此为基础, 在多介质拉氏流体动力学程序平 台上, 研制了喷射混合数值模拟程序, 实现了初始喷射 颗粒引入、气粒两相耦合计算、颗粒气动破碎、颗粒 间以及颗粒与壁面碰撞等功能 ${ }^{[40,57]}$.

使用该程序，首先开展了平面样品微喷混合实验 数值模拟，如图11所示，获得了不同初始气压下混合 区厚度和空间密度分布等信息，与实验结果符合较好, 验证了理论模型和数值程序的适用性 ${ }^{[40]}$. 此外, 通过对 混合动力学演化过程分析，获得气动效应下混合基本 规律：(1) 喷射颗粒与气体间相对速度差引起的气动 阻力效应是形成混合层的主要机制; (2)高速运动的金 属液滴在气动阻力作用下发生气动破碎，导致颗粒尺 度明显减小, 成为影响混合状态的重要物理因素 ${ }^{[58]}$.

针对内爆压缩柱面均匀汇聚构型，开展微喷颗粒 对气体反馈效应研究(双向耦合)，与不考虑微喷颗粒 对气体反馈(单向耦合)数值模拟结果(图12)对比发现: 颗粒反馈将导致气体激波提速，以及引起气区流场物 理量分布形态发生改变; 对于汇聚构型装置，几何收 缩将放大颗粒的反馈效应，可能对内爆压缩性能产生 重要影响, 需要引起高度关注 ${ }^{[59]}$. 数值模拟分析获得 的新的物理认识, 为深入理解微喷混合现象提供了重

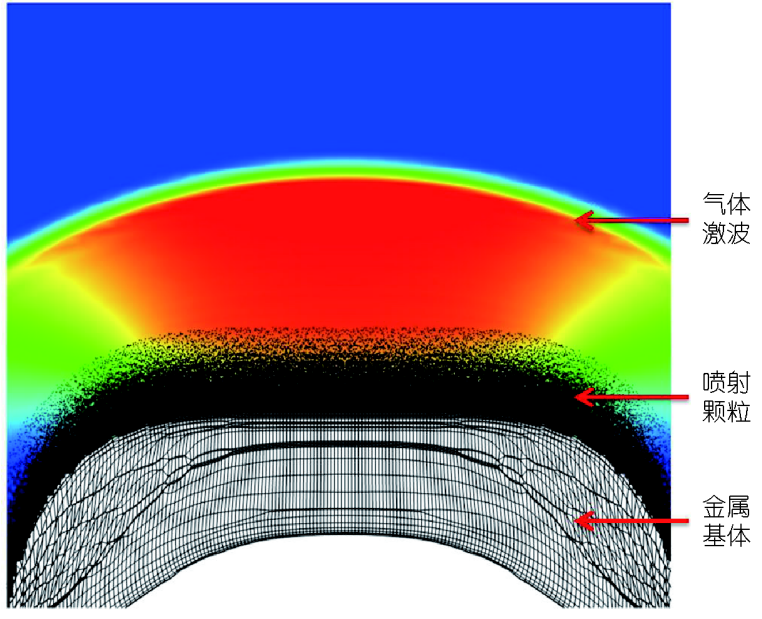

图 11 (网络版彩图)平面微喷混合数值模拟图像

Figure 11 (Color online) Snapshot of ejecta-gas mixing for planar configuration.

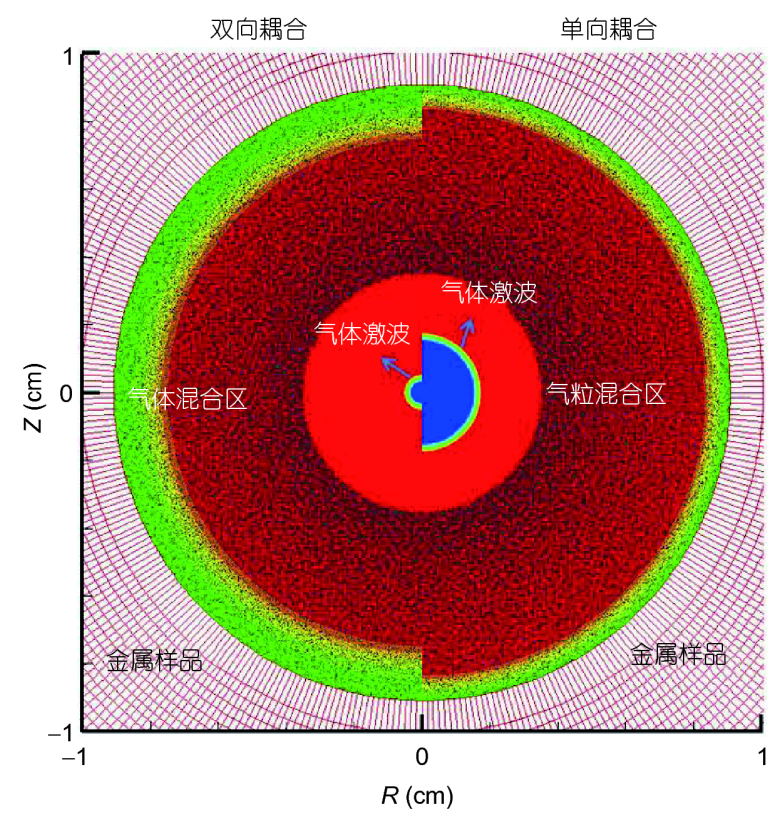

图 12 (网络版彩图)内爆微喷混合数值模拟图像

Figure 12 (Color online) Snapshot of ejecta-gas mixing for cylindrical configuration.

要理论支撑.

\section{5 总结与展望}

经过实验和理论研究者数十年的不解努力, 我们 对强冲击作用下金属微喷射及其与气体混合问题的科 学认识不断深入，逐步厘清了微喷射以及混合过程的 
主导机制和关键影响因素; 在微射流喷射理论建模方 面取得重要研究进展——建立了方波持续冲击作用下 金属宽压力区间微射流喷射理论模型.与此同时, 我们 还应该清楚认识到, 目前的微喷射与混合研究大都是 基于简化模型开展的单因素分解性实验和理论研究;
而在实际工程问题中，系统一般具有复杂的几何结构 和加载动力学状态, 时空尺度跨度很大且影响因素众 多. 如何将现有研究基础和成果应用于实际工程问题 还存在大量需要深入研究的基础性科学问题, 在面临 极大挑战的同时蕴含着重大机遇.

\section{参考文献}

1 Walsh J M, Shreffler R G, Willig F J. Limiting conditions for jet formation in high velocity collisions. J Appl Phys, 1953, 24: 349-359

2 Asay J R, Bertholf L D. Material Ejection From Shock-loaded Free Surfaces of Aluminum and Lead. Sandia Report No. SAND-76-0542, 1976

3 Asay J R. Effect of Shock Wave Rise Time on Material Ejection From Aluminum Surface. Sandia Report No. SAND-77-0731, 1977

4 Asay J R. A Model for Estimating the Effects of Surface Roughness on Mass Ejection From Shocked Materials. Sandia Report No. SAND-781256,1978

5 Vogan W S, Anderson W W, Grover M, et al. Piezoelectric characterization of ejecta from shocked tin surfaces. J Appl Phys, 2005, 98: 113508

6 Ye Y, Li Jun, Zhu P F, et al. Flash X-ray radiography for diagnosing the ejecta from shocked metal surface (in Chinese). Chin J High Press Phys, 2013, 27(3): 398-402 [叶雁, 李军, 朱鹏飞, 等. 脉冲X光照相在微物质喷射诊断中的应用. 高压物理学报, 2013, 27(3): 398-402]

7 McMillan C, Holographic Measurement of Ejecta from Shocked Metal Surface. Report: UCRL-99265, 2000

8 Ye Y, Wang W, Li Z Y, et al. High-speed photography and pulsed in-line holography diagnostics of microjet (in Chinese). Chin J High Press Phys, 2009, 23(6): 471-475 [叶雁, 汪伟, 李作友, 等. 用高速摄影和脉冲同轴全息照相联合诊断微射流. 高压物理学报, 2009, 23(6): 471-475]

9 Holtkamp D B, Clark D A, Crain M D, et al. A survey of high explosive-induced damage and spall in selected metals using proton radiography. In: Shock Compression of Condensed Matter-2003. Portland: American Institute of Physics. AIP Conf Proc, 2004, 705: 477-480

10 Han C S, Jing F Q, Ding J, et al. Study on the phenomena of the mass ejection from the free surface of aluminum at different dynamic loading rates (in Chinese). Chin J High Press Phys, 1989, 3(2): 97-106 [韩长生, 经福谦, 丁敬, 等. 不同加载速率下铝自由面微粒子喷射现象研究. 高压物理学报, 1989, 3 (2): 97-106]

11 Sorenson D S, Minich R W, Romero J L, et al. Ejecta particle size distributions for shock loaded Sn and Al metals. J Appl Phys, 2002, 92: 58305836

12 Buttler W T, Zellner M B, Olson R T, et al. Dynamic comparisons of piezoelectric ejecta diagnostics. J Appl Phys, 2007, 101: 063547

13 Andriot P, Chapron P, Olive F. Ejection of material from shocked surfaces of tin, tantalum and lead-alloys. AIP Conf Proc, 1982, 78: 505-509

14 Zellner M B, Grover M, Hammerberg J E, et al. Effects of shock-breakout pressure on ejection of micron-scale material from shocked tin surfaces. J Appl Phys, 2007, 102: 013522

15 Zellner M B, McNeil W V, Hammerberg J E, et al. Probing the underlying physics of ejecta production from shocked Sn samples. J Appl Phys, 2008, 103: 123502

16 Zhao X W, Lin X Z, Wang X J, et al. Effects of surface groove micro-structure on ejection from shocked metal surface (in Chinese). Acta Phys Sin, 2015, 64(12): 124701 [赵信文, 李欣竹, 王学军, 等. 金属表面几何缺陷微细结构对微喷射特性的影响. 物理学报, 2015, 64(12): 124701]

17 Remiot C, Chapron P, Demay B. A flash X-ray radiography diagnostic for studying surface phenomena under shock loading. AIP Conf Proc, 1994, 309(1): 1763-1766

18 Monfared S K, Oró D M, Grover M, et al. Experimental observations on the links between surface perturbation parameters and shock-induced mass ejection. J Appl Phys, 2014, 116: 063504

19 de Rességuier T, Signor L, Dragon A, et al. Experimental investigation of liquid spall in laser shock-loaded tin. J Appl Phys, 2007, 101: 013506

20 de Rességuier T, Signor L, Dragon A, et al. On the dynamic fragmentation of laser shock-melted tin. Appl Phys Lett, 2008, 92 : 131910

21 Chen Y T, Hu H B, Tang T G, et al. Experimental diagnostic of micro-spall fragments on Pb surface under intense shock (in Chinese). Sci SinPhys Mech Astron, 2012, 42: 1076-1085 [陈永涛, 胡海波, 汤铁刚, 等. 冲击加载下铅样品表面微层裂现象诊断, 中国科学: 物理学 力学 天 文学, 2012, 42: 1076-1085]

22 Loison D, de Resseguier T, Dragon A. Micro-tomography to characterize size distribution of fragments created by laser shock-induced microspallation of metallic sample. AMM, 2014, 566: 225-231 
23 Xin J, He W, Shao J, et al. Experimental investigation of fragments recovered from a laser shock-loaded tin. J Phys D-Appl Phys, 2014, 47: 325304

24 Signor L, de Rességuier T, Dragon A, et al. Investigation of fragments size resulting from dynamic fragmentation in melted state of laser shockloaded tin. Int J Impact Eng, 2010, 37: 887-900

25 Elias P, Chapron P, Mondot M. Experiment study of the slowing down of shock induced matter ejection into argon gas. Shock Compress Condens Matter, 1989: 783

26 David M O, Hammerberg J E, Buttler W T, et al. A class of ejecta transport test problems. AIP Conf Proc, 2012, 1426(1): 1351-1354

27 Ogorodnikov V A, Mikhălov A L, Burtsev V V, et al. Detecting the ejection of particles from the free surface of a shock-loaded sample. J Exp Theor Phys, 2009, 109: 530-535

28 Sorenson D S, Pazuchanics P, Johnson R P, et al. Ejecta particle-size measurements in vacuum and helium gas using ultraviolet in-line Fraunhofer holography. Report No. LA-UR-14-24722, 2014

29 Chen J, Jing F Q, Zhang J L, et al. Dynamics simulation of ejection of metal under a shock wave. J Phys-Condens Matter, 2002, 14: 10833-10837

30 Germann T C, Hammerberg J E, Holian B L. Large-scale molecular dynamics simulations of ejecta formation in copper. AIP Conf Proc, 2003, 706: 285

31 Shao J L, Wang P, He A M, et al. Atomistic simulations of shock-induced microjet from a grooved aluminium surface. J Appl Phys, 2013, 113: 153501

32 Shao J L, Wang P, He A M, et al. Molecular dynamics study on the failure modes of aluminium under decaying shock loading. J Appl Phys, 2013, 113: 163507

33 Durand O, Soulard L. Large-scale molecular dynamics study of jet breakup and ejecta production from shock-loaded copper with a hybrid method. J Appl Phys, 2012, 111: 044901

34 Xiang M, Hu H, Chen J, et al. Molecular dynamics simulations of micro-spallation of single crystal lead. Model Simul Mater Sci Eng, 2013, 21: 055005

35 He A M, Wang P, Shao J L, et al. Molecular dynamics simulations of jet breakup and ejecta production from a grooved Cu surface under shock loading. Chin Phys B, 2014, 23: 047102

36 Buttler W T, Oró D M, Preston D L, et al. Unstable Richtmyer-Meshkov growth of solid and liquid metals in vacuum. J Fluid Mech, 2012, 703: $60-84$

37 Dimonte G, Terrones G, Cherne F J, et al. Ejecta source model based on the nonlinear Richtmyer-Meshkov instability. J Appl Phys, 2013, 113: 024905

38 Cherne F J, Hammerberg J E, Andrews M J, et al. On shock driven jetting of liquid from non-sinusoidal surfaces into a vacuum. J Appl Phys, 2015, 118: 185901

39 Fung J, Harrison A K, Chitanvis S, et al. Ejecta source and transport modeling in the FLAG hydrocode. Comput Fluids, 2013, 83: 177-186

40 Wang P, Sun H Q, Shao J L, et al. Numerical simulation on mixing process of ejecta and gas (in Chinese). Acta Phys Sin, 2012, 61 : 234703 [王裴, 孙海权, 邵建立, 等. 微喷颗粒与气体混合过程的数值模拟研究. 物理学报, 2012, 61: 234703]

41 Wang P, Qin C S, Zhang S D, et al. Simulated microjet from free surface of aluminum using smoothed particle hydrodynamics (in Chinese). Chin J High Press Phys, 2004, 18(02): 149-156 [王裴, 秦承森, 张树道, 等. SPH方法对金属表面微射流的数值模拟. 高压物理学报, 2004, 18(02): 149-156]

42 Wang P, Shao J L, Qin C S. Effect of loading-wave-front width on micro-jet from aluminum surface (in Chinese). Acta Phys Sin, 2009, 58(2): 1064-1070. [王裴, 邵建立, 秦承森. 加载波前沿宽度对铝表面微射流的影响. 物理学报, 2009, 58(2): 1064-1070]

43 Wang P, Shao J L, Qin C S. Groove angle effect on micro-jet from shocked metal surface (in Chinese). Acta Phys Sin, 2012, 61(23): 234701 [王 裴, 邵建立, 秦承森. 沟槽角度对金属表面微射流性质的影响, 物理学报, 2012, 61(23): 234701]

44 Liu J, Feng Q J, Zhou H B. Simulation study of interface instability in metals driven by cylindrical implosion (in Chinese). Acta Phys Sin, 2014, 63: 155201 [刘军, 冯其京, 周海兵. 柱面内爆驱动金属界面不稳定性的数值模拟研究. 物理学报, 2014, 63: 155201]

45 Dimonte G, Terrones G, Cherne F J, et al. Use of the Richtmyer-Meshkov instability to infer yield stress at high-energy densities. Phys Rev Lett, 2011, 107: 264502

46 Piriz A R, López Cela J J, Tahir N A, et al. Richtmyer-Meshkov instability in elastic-plastic media. Phys Rev E, 2008, 78: 056401

47 Mikaelian K O. Shock-induced interface instability in viscous fluids and metals. Phys Rev E, 2013, 87: 031003 
He A M, Wang P, Liu J. Theoretical model of the total mass of ejecta from unmelted metals. In: Gervasi O, Murgante B, Misra S, et al., eds. Computational Science and Its Applications-ICCSA 2017. Lecture Notes in Computer Science, 2017, 10409: 693-700

49 He A M, Liu J, Shao J L, et al. Theoretical ejeacta model based on Richtmyer-Meshkov instability for elastic-plastic solid (in Chinese). Chin J Comp Phys, in press [何安民, 刘军, 邵建立, 等. 基于强度介质Richtmyer-Meshkov不稳定性理论的微缺陷喷射模型. 计算物理, 待发表]

50 Durand O, Soulard L. Power law and exponential ejecta size distributions from the dynamic fragmentation of shock-loaded Cu and Sn metals under melt conditions. J Appl Phys, 2013, 114: 194902, arXiv: 1310.6999

51 He A M, Wang P, Shao J L. Molecular dynamics simulations of ejecta size distributions for shock-loaded $\mathrm{Cu}$ with a wedged surface groove. Comput Mater Sci, 2015, 98: 271-277

52 He A M, Wang P, Shao J L. Statistically heterogeneous size distribution of ejecta from shock-loaded Cu with a wedged surface groove. Model Simul Mater Sci Eng, 2016, 24: 025002

53 He W, Xin J, Zhao Y, et al. Fragment size distribution statistics in dynamic fragmentation of laser shock-loaded tin. AIP Adv, 2017, 7: 065306

54 He A M, Wang Pei, Shao J L. Heterogeneous fragmentation of metallic liquid microsheet with high velocity gradient. Chin Phys B, 2016, 25: 017102

55 Liu W B, Ma D J, He A M, et al. Ejecta from periodic grooved Sn surface under unsupported shocks. Chin Phys B, 2018, 27: 016202

56 Shao J L, Wang P, He A M. Influence of shock pressure and profile on the microjetting from a grooved Pb surface. Model Simul Mater Sci Eng, 2017, 25: 015011

57 Sun H Q, Wang P. Research of shock induced matter ejection into gas (in Chinese). Chin J Comp Mech, 2011, 28(B04): 90-92 [孙海权, 王裴. 微 喷混合问题的初步研究. 计算力学学报, 2011, 28(B04): 90-92]

58 Sun H Q, Wang P, Chen D W, et al. Breakup of particles in ejecta mixing (in Chinese). Explosion Shock Waves, 2014, 34(04): 392-396 [孙海权, 王裴, 陈大伟, 等. 微喷混合中的颗粒破碎效应. 爆炸与冲击, 2014, 34(04): 392-396]

59 Chen D W, Sun H Q, Wang P, et al. Numerical investigation on the influence of gas-particle two-way coupling to the shock fluid in the twodimensional Lagrangian framework (in Chinese). Acta Phys Sin, 2016, 65(8): 084703 [陈大伟, 孙海权, 王裴, 等. 二维拉格朗日坐标系下气粒 混合双向耦合对激波流场影响的计算. 物理学报, 2016, 65(8): 084703] 


\title{
Numerical and theoretical investigations of shock-induced material ejection and ejecta-gas mixing
}

\author{
WANG Pei ${ }^{1 *}$, HE AnMin ${ }^{1}$, SHAO JianLi ${ }^{2}$, SUN HaiQuan ${ }^{1}$, CHEN DaWei ${ }^{1}$, \\ LIU WenBin ${ }^{1,3} \&$ LIU Jun ${ }^{1}$ \\ ${ }^{1}$ Institute of Applied Physics and Computational Mathematics, Beijing 100094, China; \\ ${ }^{2}$ Key Laboratory of Explosion Science and Technology, Beijing Institute of Technology, Beijing 100081, China; \\ ${ }^{3}$ Graduate School of China Academy of Engineering Physics, Beijing 100088, China
}

\begin{abstract}
When a strong shock wave releases from material surface, material ejection usually takes place where a great amount of high-speed particles can be ejected from the material surface. This phenomenon is called micro-ejection and the ejected particles is named as ejecta. In gas environment, the interaction between ejecta and gas can lead to the formation of a layer of gas-ejecta mixture and this process is called mixing. Micro-ejection and mixing are of great importance in many fields such as inertial confinement fusion and implosive dynamics, and have attracted intense research activities both experimentally and theoretically in the past several decades. The author and collaborators have been studying the microejection and mixing phenomena for years, and some valuable achievements have been obtained. In the present review, we introduce some of our theoretical works on the two main mechanisms of micro-ejection (i.e., micro-jetting and microspallation) as well as the gas-ejecta mixing process, including mechanisms of micro-jetting and micro-spallation and their dominant factors, modeling of micro-jetting based on the Richtmyer-Meshkov theory in elastic-plastic solids, and some dynamic characteristics of mixing.
\end{abstract}

micro-ejection, mixing, micro-jetting, micro-spallation

PACS: $47.15 . \mathrm{Uv}, 62.50 . \mathrm{Ef}, 47.11 .-\mathrm{j}$

doi: 10.1360/SSPMA2018-00116 